\title{
Editorial: advances in neuro-oncology and clinical treatment-from ASNO 2019
}

\author{
Pin-Yuan Chen ${ }^{1,2,3} \cdot$ Kuo-Chen Wei ${ }^{2,3}$
}

Received: 3 November 2019 / Accepted: 12 November 2019 / Published online: 4 February 2020

๑) Springer Science+Business Media, LLC, part of Springer Nature 2019

\begin{abstract}
The 16th Annual Meeting of the Asian Society for Neuro-Oncology was successfully held in Taipei, September 26-29. The diverse, in-depth experiences sharing in the conference contributed the fruitful result. Here we selected several important manuscripts to represent the advance of neuro-oncology and clinical treatment in Asia.
\end{abstract}

Keywords ASNO2019 $\cdot$ Neuro-oncology $\cdot$ Treatment

Asia is a quickly developing area and so is the field of neurooncology. During the 16th Annual Meeting of the Asian Society for Neuro-Oncology in Taipei we covered many aspects of neuro-oncology, including clinical treatment and molecular mechanisms, adult and pediatric categories, the subspecialty of spinal tumors, and the application of artificial intelligence to advanced therapies such as immunotherapies and tumor treating fields (TTF). Here, we have selected several manuscripts to represent the great diversity of this meeting.

Surgical excision followed by chemoradiation therapy and adjuvant chemotherapy has been the standard treatment for glioblastoma (GBM) since 2005, and no other therapies have truly improved the median overall survival (OS). Tumor treating fields were approved by the FDA for recurrent GBM in 2011, and the indication was extended to newly diagnosed GBM in 2015. Combined with temozolomide (TMZ) treatment in the EF-14 trial, TTF was shown to improve the median OS from 16 to 20.9 months [1]. Kim et al. [2] analyzed the Korean subgroup in the EF-14 trial, and reported 1- and 2-year survival rates of $95.6 \%$ versus $73 \%$ and $60 \%$ versus $30 \%$ with TTF/TMZ and TMZ alone,

Pin-Yuan Chen

pinyuanc@gmail.com

1 Department of Neurosurgery, Chang Gung Memorial Hospital at Keelung, Keelung, Taiwan

2 Department of Neurosurgery, Chang Gung Memorial Hospital at Linkou, Taoyüan, Taiwan

3 School of Medicine, Chang Gung University, Taoyüan, Taiwan respectively. Moreover, the median OS was 27.2 months (95\% CI 21-NA) with TTF/TMZ versus 15.2 months (95\% CI 7.5-24.1; HR 0.27, $p=0.01$ ) with TMZ alone. However, this therapy has not been available in Asia apart from Japan until this year, when regular clinical use was started in Hong Kong. We hope this treatment will be approved soon for use in other Asian countries so that more Asian patients can benefit from the positive therapeutic effects.

Immunotherapies, and especially immune checkpoint inhibitors, have shown great success in many malignancies, including melanoma, lung, breast, head and neck, and colon cancer, but not in glioma. The unique features of the blood brain barrier, different immune cell components and complex tumor microenvironment all contribute to this failure. Modulation of tumor-associated immune cells may be key to overcoming these problems. Hsu et al. [3] reported that the combination of rapamycin and hydroxychloroquine could sensitize glioma xenografts to anti-PD1 treatment by altering macrophages into the M1 type. More experimental research is needed to elucidate the complex interactions between drugs, tumor cells, macrophages/microglia, and T lymphocytes so that immunotherapy can be applied for glioma.

Somatic mutations of the isocitrate dehydrogenase 1 gene (IDH1 m) are a pathological hallmark of lower grade glioma and secondary GBM. The role of its neo metabolic product, (d)-2-hydroxyglutarate (2HG), is believed to promote glioma initiation and progression through epigenetic and metabolic reprogramming [4]. However, despite this postulated molecular mechanism, patients with IDH1 $\mathrm{m}$ have been reported to have a longer OS than patients without IDH1 $\mathrm{m}$. Tiburcio et al. [5] reported that 3D-cultured $\mathrm{IDH} 1^{\mathrm{R} 132 \mathrm{H}}$-hemizygous 
cells exhibited the glioblastoma mesenchymal signature, but that IDH $1^{\mathrm{R} 132 \mathrm{H}}$-heterozygous cells did not. Interestingly, an IDH1 inhibitor, AGI-5198, promoted the growth of IDH $1^{\mathrm{R} 132 \mathrm{H}}$-heterozygous cells, the expression of neural stem-cell marker genes, and redox homeostasis independently of GLUD2. This study may partially explain how simple target therapy can cause complex and unexpected responses.

Stereotactic radiosurgery (SRS) plays a definite role in the local control of brain metastasis. Guidelines from the American Congress of Neurological Surgeons recommend SRS for patients with multiple brain metastases, and even for more than four lesions as long as their cumulative volume is under $7 \mathrm{~mL}$ [6]. Huang et al. [7] reported that several radiomic features including shape flatness, skewness, Gray Level Co-occurrence Matrix (GLCM) cluster shade and GLCM correlation could predict the response of metastatic brain tumors to Gamma Knife radiosurgery in a group of lung cancer patients. Whether the local response to SRS was influenced by the patients' general medical condition and concomitant target or immunotherapies was uncertain. Using artificial intelligence to analyze clinical characteristics, molecular pathological factors and imaging features will be valuable to improve this therapy.

We would like to express our gratitude to everyone who contributed to this meeting and these special issues. It is our sincere hope that the exchange of knowledge and professional experiences along with the strengthening of friendships will not only be of great benefit to ourselves, but ultimately to our patients and their families.

\section{Compliance with ethical standards}

Conflict of interest All authors declare that they have no conflict of interest.
Ethical approval This article does not contain any studies with human participants or animals performed by any of the authors.

\section{References}

1. Stupp R, Taillibert S, Kanner AA, Kesari S, Steinberg DM, Toms SA, Taylor LP, Lieberman F, Silvani A, Fink KL, Barnett GH, Zhu J-J, Henson JW, Engelhard HH, Chen TC, Tran DD, Sroubek J, Tran ND, Hottinger AF, Landolfi J, Desai R, Caroli M, Kew Y, Honnorat J, Idbaih A, Kirson ED, Weinberg U, Palti Y, Hegi ME, Ram Z (2015) Maintenance therapy with tumor-treating fields plus temozolomide vs temozolomide alone for glioblastoma. JAMA 314:25-35. https://doi.org/10.1001/jama.2015.16669

2. Kim CY, Paek SH, Nam DH, Chang JH, Hong YK, Kim JH, Kim OL, Kim SH (2019) Tumor treating fields plus temozolomide for newly diagnosed glioblastoma: a sub-group analysis of Korean patients in the EF-14 phase 3 trial. J Neurooncol. https://doi. org/10.1007/s11060-019-03361-2

3. Hsu SPC, Chen YC, Chiang HC, Huang YC, Huang CC, Wang HE, Wang YS, Chi KH (2019) Rapamycin and hydroxychloroquine combination alters macrophage polarization and sensitizes glioblastoma to immune check point inhibitors. J Neurooncol. https://doi.org/10.1007/s11060-019-03360-3

4. Huang LE (2019) Friend or foe-IDH1 mutations in glioma 10 years on. Carcinogenesis 1-9

5. Tiburcio PDB, Gillespie DL, Jensen RL, Huang LE (2019) Extracellular glutamate and IDH1R132H inhibitor promote glioma growth by boosting redox potential. J Neurooncol. https://doi. org/10.1007/s11060-019-03359-w

6. Graber JJ, Cobbs CS, Olson JJ (2019) Congress of neurological surgeons systematic review and evidence-based guidelines on the use of stereotactic radiosurgery in the treatment of adults with metastatic brain tumors. Neurosurgery 84(3):E168-E170

7. Huang CY, Lee CC, Yang HC, Lin CJ, Wu HM, Chung WY, Shiau CY, Guo WY, Pan DHC, Peng SJ (2019) Radiomics as prognostic factor in brain metastases treated with Gamma Knife radiosurgery. J Neurooncol. https://doi.org/10.1007/s11060-019-03343-4

Publisher's Note Springer Nature remains neutral with regard to jurisdictional claims in published maps and institutional affiliations. 